\title{
On Hilbert type inequalities
}

\section{Chang-Jian Zhao $^{1 *}$ and Wing-Sum Cheung ${ }^{2}$}

"Correspondence:

chjzhao@yahoo.com.cn;

chjzhao@163.com

1 Department of Mathematics, China

Jiliang University, Hangzhou

310018 , P.R. China

Full list of author information is

available at the end of the article

\begin{abstract}
In the present paper we establish new inequalities similar to the extensions of Hilbert's double-series inequality and also give their integral analogues. Our results provide some new estimates to these types of inequalities.
\end{abstract}

MSC: $26 \mathrm{D} 15$

Keywords: Hilbert's inequality; Pachpatte's inequality; Hölder's inequality

\section{Introduction}

In recent years several authors have given considerable attention to Hilbert's double-series inequality together with its integral version, inverse version, and various generalizations (see [1-9]). In this paper, we establish multivariable sum inequalities for the extensions of Hilbert's inequality and also obtain their integral forms. Our results provide some new estimates to these types of inequalities.

The well-known classical extension of Hilbert's double-series theorem can be stated as follows [10, p.253].

Theorem A If $p_{1}, p_{2}>1$ are real numbers such that $\frac{1}{p_{1}}+\frac{1}{p_{2}} \geq 1$ and $0<\lambda=2-\frac{1}{p_{1}}-\frac{1}{p_{2}}=$ $\frac{1}{q_{1}}+\frac{1}{q_{2}} \leq 1$, where, as usual, $q_{1}$ and $q_{2}$ are the conjugate exponents of $p_{1}$ and $p_{2}$ respectively, then

$$
\sum_{m=1}^{\infty} \sum_{n=1}^{\infty} \frac{a_{m} b_{n}}{(m+n)^{\lambda}} \leq K\left(\sum_{m=1}^{\infty} a_{m}^{p}\right)^{1 / p_{1}}\left(\sum_{n=1}^{\infty} b_{n}^{q}\right)^{1 / p_{2}},
$$

where $K=K\left(p_{1}, p_{2}\right)$ depends on $p_{1}$ and $p_{2}$ only.

In 2000, Pachpatte [11] established a new inequality similar to inequality (1.1) as follows:

Theorem $\mathbf{A}^{\prime}$ Let $p, q, a(s), b(t), a(0), b(0), \nabla a(s)$ and $\nabla b(t)$ be as in [11], then

$$
\begin{aligned}
\sum_{s=1}^{m} \sum_{t=1}^{n} \frac{|a(s)||b(t)|}{q s^{p-1}+p t^{q-1}} \leq & \frac{1}{p q} m^{(p-1) / p} n^{(q-1) / q}\left(\sum_{s=1}^{m}(m-s+1)|\nabla a(s)|^{p}\right)^{1 / p} \\
& \times\left(\sum_{t=1}^{n}(n-t+1)|\nabla b(t)|^{q}\right)^{1 / q} .
\end{aligned}
$$

The integral analogue of inequality (1.1) is as follows [10, p.254].

\section{Springer}

(c) 2012 Zhao and Cheung; licensee Springer. This is an Open Access article distributed under the terms of the Creative Commons Attribution License (http://creativecommons.org/licenses/by/2.0), which permits unrestricted use, distribution, and reproduction in any medium, provided the original work is properly cited. 
Theorem B Let $p, q, p^{\prime}, q^{\prime}$ and $\lambda$ be as in Theorem A. Iff $\in L^{p}(0, \infty)$ and $g \in L^{q}(0, \infty)$, then

$$
\int_{0}^{\infty} \int_{0}^{\infty} \frac{f(x) g(x)}{(x+y)^{\lambda}} d x d y \leq K\left(\int_{0}^{\infty} f^{p}(x) d x\right)^{1 / p}\left(\int_{0}^{\infty} g^{q}(y) d y\right)^{1 / q}
$$

where $K=K(p, q)$ depends on $p$ and $q$ only.

In [11], Pachpatte also established a similar version of inequality (1.3) as follows.

Theorem B' Let $p, q, f(s), g(t), f(0), g(0), f^{\prime}(s)$ and $g^{\prime}(t)$ be as in [11], then

$$
\begin{aligned}
& \int_{0}^{x} \int_{0}^{y} \frac{|f(s)||g(t)|}{q s^{p-1}+p t^{q-1}} d t d s \\
& \quad \leq \frac{1}{p q} x^{(p-1) / p} y^{(q-1) / q}\left(\int_{0}^{x}(x-s)\left|f^{\prime}(s)\right|^{p} d s\right)^{1 / p}\left(\int_{0}^{y}(y-t)\left|g^{\prime}(t)\right|^{q} d t\right)^{1 / q} .
\end{aligned}
$$

In the present paper we establish some new inequalities similar to Theorems A, $\mathrm{A}^{\prime}, \mathrm{B}$ and $\mathrm{B}^{\prime}$. Our results provide some new estimates to these types of inequalities.

\section{Statement of results}

Our main results are given in the following theorems.

Theorem 2.1 Let $p_{i}>1$ be constants and $\frac{1}{p_{i}}+\frac{1}{q_{i}}=1$. Let $a_{i}\left(s_{1 i}, \ldots, s_{n i}\right)$ be real-valued functions defined for $s_{j i}=1,2, \ldots, m_{j i}$, where $m_{j i}(i, j=1,2, \ldots, n)$ are natural numbers. For convenience, we write $a_{i}(0, \ldots, 0)=0$ and $a_{i}\left(0, s_{2 i}, \ldots, s_{n i}\right)=a_{i}\left(s_{1 i}, 0, s_{3 i}, \ldots, s_{n i}\right)=$ $\cdots=a_{i}\left(s_{1 i}, \ldots, s_{n-1, i}, 0\right)=0$. Define the operators $\nabla_{i}$ by $\nabla_{i} a_{i}\left(s_{1 i}, \ldots, s_{n i}\right)=a_{i}\left(s_{1 i}, \ldots, s_{n i}\right)-$ $a_{i}\left(s_{1 i}, \ldots, s_{i-1, i}, s_{i i}-1, s_{i+1, i}, \ldots, s_{n i}\right)$ for any function $a_{i}\left(s_{1 i}, \ldots, s_{n i}\right)$. Then

$$
\begin{aligned}
& \sum_{s_{11}=1}^{m_{11}} \cdots \sum_{s_{n 1}=1}^{m_{n 1}} \sum_{s_{12}=1}^{m_{12}} \cdots \sum_{s_{n 2}=1}^{m_{n 2}} \cdots \sum_{s_{1 n}=1}^{m_{1 n}} \cdots \sum_{s_{n n}=1}^{m_{n n}} \frac{\prod_{i=1}^{n}\left|a_{i}\left(s_{1 i}, \ldots, s_{n i}\right)\right|}{\left(\sum_{i=1}^{n}\left(s_{1 i} \cdots s_{n i}\right) / q_{i}\right)^{\sum_{i=1}^{n} 1 / q_{i}}} \\
& \quad \leq M \prod_{i=1}^{n}\left(\sum_{s_{n i}=1}^{m_{n i}} \cdots \sum_{s_{1 i}=1}^{m_{1 i}} \prod_{j=1}^{n}\left(m_{j i}-s_{j i}+1\right)\left|\nabla_{n} \cdots \nabla_{1} a_{i}\left(s_{1 i}, \ldots, s_{n i}\right)\right|^{p_{i}}\right)^{1 / p_{i}}
\end{aligned}
$$

where

$$
M=M\left(m_{1 i}, \ldots, m_{n i}\right)=\left(n-\sum_{i=1}^{n} 1 / p_{i}\right)^{\sum_{i=1}^{n} 1 / p_{i}-n} \prod_{i=1}^{n}\left(m_{1 i} \cdots m_{n i}\right)^{1 / q_{i}} .
$$

Remark 2.1 Let $a_{i}\left(s_{1 i}, \ldots, s_{n i}\right)$ change to $a_{i}\left(s_{i}\right)$ in Theorem 2.1 and in view of $a_{i}(0)=0$ and $\nabla a_{i}\left(s_{i}\right)=a_{i}\left(s_{i}\right)-a_{i}\left(s_{i}-1\right)$ for any function $a_{i}\left(s_{i}\right), i=1,2, \ldots, n$, then

$$
\sum_{s_{1}=1}^{m_{1}} \sum_{s_{2}=1}^{m_{2}} \cdots \sum_{s_{n}=1}^{m_{n}} \frac{\prod_{i=1}^{n}\left|a_{i}\left(s_{i}\right)\right|}{\left(\sum_{i=1}^{n} s_{i} / q_{i}\right)^{\sum_{i=1}^{n} 1 / q_{i}}} \leq \bar{M} \prod_{i=1}^{n}\left(\sum_{s_{i}=1}^{m_{i}}\left(m_{i}-s_{i}+1\right)\left|\nabla a_{i}\left(s_{i}\right)\right|^{p_{i}}\right)^{1 / p_{i}},
$$

where

$$
\bar{M}=\bar{M}\left(m_{1}, \ldots, m_{n}\right)=\left(n-\sum_{i=1}^{n} \frac{1}{p_{i}}\right)^{\sum_{i=1}^{n} 1 / p_{i}-n} \cdot \prod_{i=1}^{n} m_{i}^{1 / q_{i}} .
$$


Remark 2.2 Taking for $n=2$ in Remark 2.1. If $p_{1}, p_{2}>1$ satisfy $\frac{1}{p_{1}}+\frac{1}{p_{2}} \geq 1$ and $0<\lambda=$ $2-\frac{1}{p_{1}}-\frac{1}{p_{2}}=\frac{1}{q_{1}}+\frac{1}{q_{2}} \leq 1$, then inequality $(2.2)$ reduces to

$$
\begin{aligned}
& \sum_{s_{1}=1}^{m_{1}} \sum_{s_{2}=1}^{m_{2}} \frac{\left|a_{1}\left(s_{1}\right)\right|\left|a_{2}\left(s_{2}\right)\right|}{\left(q_{2} s_{1}+q_{1} s_{2}\right)^{\lambda}} \\
& \leq \frac{1}{\left(\lambda q_{1} q_{2}\right)^{\lambda}} m_{1}^{1 / q_{1}} m_{2}^{1 / q_{2}}\left(\sum_{s_{1}=1}^{m_{1}}\left(m_{1}-s_{1}+1\right)\left|\nabla a_{1}\left(s_{1}\right)\right|^{p_{1}}\right)^{1 / p_{1}} \\
& \quad \times\left(\sum_{s_{2}=1}^{m_{2}}\left(m_{2}-s_{2}+1\right)\left|\nabla a_{2}\left(s_{2}\right)\right|^{p_{2}}\right)^{1 / p_{2}}
\end{aligned}
$$

which is an interesting variation of inequality (1.1).

On the other hand, if $\lambda=1$, then $\frac{1}{p_{1}}+\frac{1}{p_{2}}=\frac{1}{q_{1}}+\frac{1}{q_{2}}=1$ and so $p_{1}=q_{2}, p_{2}=q_{1}$. In this case inequality (2.3) reduces to

$$
\begin{aligned}
& \sum_{s_{1}=1}^{m_{1}} \sum_{s_{2}=1}^{m_{2}} \frac{\left|a_{1}\left(s_{1}\right)\right|\left|a_{2}\left(s_{2}\right)\right|}{p_{1} s_{1}+q_{1} s_{2}} \\
& \leq \frac{1}{p_{1} q_{1}} m_{1}^{\left(p_{1}-1\right) / p_{1}} m_{2}^{\left(q_{1}-1\right) / q_{1}}\left(\sum_{s_{1}=1}^{m_{1}}\left(m_{1}-s_{1}+1\right)\left|\nabla a_{1}\left(s_{1}\right)\right|^{p_{1}}\right)^{1 / p_{1}} \\
& \quad \times\left(\sum_{s_{2}=1}^{m_{2}}\left(m_{2}-s_{2}+1\right)\left|\nabla a_{2}\left(s_{2}\right)\right|^{q_{1}}\right)^{1 / q_{1}} .
\end{aligned}
$$

This is just a similar version of inequality (1.2) in Theorem $\mathrm{A}^{\prime}$.

Theorem 2.2 Let $p_{i}>1$ be constants and $\frac{1}{p_{i}}+\frac{1}{q_{i}}=1$. Let $f_{i}\left(\tau_{1 i}, \ldots, \tau_{n i}\right)$ be real-valued $n t h$ differentiable functions defined on $\left[0, x_{1 i}\right) \times \cdots \times\left[0, x_{n i}\right)$, where $0 \leq x_{j i} \leq t_{j i}, t_{j i} \in(0, \infty)$ and $i, j=1,2, \ldots, n$. Suppose

$$
f_{i}\left(x_{1 i}, \ldots, x_{n i}\right)=\int_{0}^{x_{1 i}} \cdots \int_{0}^{x_{n i}} \frac{\partial^{n}}{\partial \tau_{1 i} \cdots \partial \tau_{n i}} f_{i}\left(\tau_{1 i}, \ldots, \tau_{n i}\right) d \tau_{1 i} \cdots d \tau_{n i},
$$

then

$$
\begin{aligned}
\int_{0}^{t_{11}} & \cdots \int_{0}^{t_{n 1}} \int_{0}^{t_{12}} \cdots \int_{0}^{t_{n 2}} \cdots \int_{0}^{t_{1 n}} \cdots \int_{0}^{t_{n n}} \\
& \frac{\prod_{i=1}^{n}\left(\int_{0}^{x_{1 i}} \cdots \int_{0}^{x_{n i}}\left|\frac{\partial^{n}}{\partial \tau_{1 i} \cdots \partial \tau_{n l}} f_{i}\left(\tau_{1 i}, \ldots, \tau_{n i}\right)\right|^{p_{i}} d \tau_{1 i} \cdots d \tau_{n i}\right)^{1 / p_{i}}}{\left(\sum_{i=1}^{n}\left(x_{1 i} \cdots x_{n i}\right) / q_{i}\right)^{\sum_{i=1}^{n} 1 / q_{i}}} \\
& d x_{11} \cdots d x_{n 1} d x_{12} \cdots d x_{n 2} \cdots d x_{1 n} \cdots d x_{n n} \\
\leq & N \prod_{i=1}^{n}\left(\int_{0}^{t_{1 i}} \cdots \int_{0}^{t_{n i}} \prod_{j=1}^{n}\left(t_{j i}-x_{j i}\right)\right. \\
& \left.\times\left|\frac{\partial^{n}}{\partial x_{1 i} \cdots \partial x_{n i}} f_{i}\left(x_{1 i}, \ldots, x_{n i}\right)\right|^{p_{i}} d x_{1 i} \cdots d x_{n i}\right)^{1 / p_{i}},
\end{aligned}
$$


where

$$
N=N\left(t_{1 i}, \ldots, t_{n i}\right)=\left(n-\sum_{i=1}^{n} \frac{1}{p_{i}}\right)^{\sum_{i=1}^{n} 1 / p_{i}-n} \cdot \prod_{i=1}^{n}\left(t_{1 i} \cdots t_{n i}\right)^{1 / q_{i}}
$$

Remark 2.3 Let $f_{i}\left(x_{1 i}, \ldots, x_{n i}\right)$ change to $f_{i}\left(s_{i}\right)$ in Theorem 2.2 and in view of $f_{i}(0)=0, i=$ $1,2, \ldots, n$, then

$$
\begin{gathered}
\int_{0}^{x_{1}} \ldots \int_{0}^{x_{n}} \frac{\prod_{i=1}^{n}\left|f\left(s_{i}\right)\right|}{\left(\sum_{i=1}^{n} s_{i} / q_{i}\right)^{\sum_{i=1}^{n} 1 / q_{i}}} d s_{n} \cdots d s_{1} \\
\leq \bar{N} \prod_{i=1}^{n}\left(\int_{0}^{x_{i}}\left(x_{i}-s_{i}\right)\left|f_{i}^{\prime}\left(s_{i}\right)\right|^{p_{i}} d s_{i}\right)^{1 / p_{i}},
\end{gathered}
$$

where

$$
\bar{N}=\bar{N}\left(x_{1}, \ldots, x_{n}\right)=\left(n-\sum_{i=1}^{n} \frac{1}{p_{i}}\right)^{\sum_{i=1}^{n} 1 / p_{i}-n} \cdot \prod_{i=1}^{n} x_{i}^{1 / q_{i}} .
$$

Remark 2.4 Taking for $n=2$ in Remark 2.3, if $p_{1}, p_{2}>1$ are such that $\frac{1}{p_{1}}+\frac{1}{p_{2}} \geq 1$ and $0<\lambda=2-\frac{1}{p_{1}}-\frac{1}{p_{2}}=\frac{1}{q_{1}}+\frac{1}{q_{2}} \leq 1$, inequality $(2.5)$ reduces to

$$
\begin{aligned}
& \int_{0}^{x_{1}} \int_{0}^{x_{2}} \frac{\left|f_{1}\left(s_{1}\right)\right|\left|f_{2}\left(s_{2}\right)\right|}{\left(q_{2} s_{1}+q_{1} s_{2}\right)^{\lambda}} d s_{2} d s_{1} \\
& \leq \frac{1}{\left(\lambda q_{1} q_{2}\right)^{\lambda}} x_{1}^{1 / q_{1}} x_{2}^{1 / q_{2}}\left(\int_{0}^{x_{1}}\left(x_{1}-s_{1}\right)\left|f_{1}^{\prime}\left(s_{1}\right)\right|^{p_{1}} d s_{1}\right)^{1 / p_{1}} \\
& \quad \times\left(\int_{0}^{x_{2}}\left(x_{2}-s_{2}\right)\left|f_{2}^{\prime}\left(s_{2}\right)\right|^{p_{2}} d s_{2}\right)^{1 / p_{2}},
\end{aligned}
$$

which is an interesting variation of inequality (1.3).

On the other hand, if $\lambda=1$, then $\frac{1}{p_{1}}+\frac{1}{p_{2}}=\frac{1}{q_{1}}+\frac{1}{q_{2}}=1$ and so $p_{1}=q_{2}, p_{2}=q_{1}$. In this case inequality (2.6) reduces to

$$
\begin{aligned}
& \int_{0}^{x_{1}} \int_{0}^{x_{2}} \frac{\left|f_{1}\left(s_{1}\right)\right|\left|f_{2}\left(s_{2}\right)\right|}{p_{1} s_{1}+q_{1} s_{2}} \\
& \leq \frac{h_{1} h_{2}}{p_{1} q_{1}} x_{1}^{\left(p_{1}-1\right) / p_{1}} x_{2}^{\left(q_{1}-1\right) / q_{1}}\left(\int_{0}^{x_{1}}\left(x_{1}-s_{1}\right)\left|f_{1}^{\prime}\left(s_{1}\right)\right|^{p_{1}} d s_{1}\right)^{1 / p_{1}} \\
& \quad \times\left(\int_{0}^{x_{2}}\left(x_{2}-s_{2}\right)\left|f_{2}^{\prime}\left(s_{2}\right)\right|^{q_{1}} d s_{2}\right)^{1 / q_{1}} .
\end{aligned}
$$

This is just a similar version of inequality (1.4) in Theorem $\mathrm{B}^{\prime}$. 


\section{Proofs of results}

Proof of Theorem 2.1 From the hypotheses $a_{i}(0, \ldots, 0)=a_{i}\left(0, s_{2 i}, \ldots, s_{n i}\right)=a_{i}\left(s_{1 i}, 0, s_{3 i}, \ldots\right.$, $\left.s_{n i}\right)=\cdots=a_{i}\left(s_{1 i}, \ldots, s_{n-1, i}, 0\right)=0$, we have

$$
\left|a_{i}\left(s_{1 i}, \ldots, s_{n i}\right)\right| \leq \sum_{\tau_{n i}=1}^{s_{n i}} \cdots \sum_{\tau_{1 i}=1}^{s_{1 i}}\left|\nabla_{n} \cdots \nabla_{1} a_{i}\left(\tau_{1 i}, \ldots, \tau_{n i}\right)\right|
$$

From the hypotheses of Theorem 2.1 and in view of Hölder's inequality (see [10]) and inequality for mean [10], we obtain

$$
\begin{aligned}
\prod_{i=1}^{n}\left|a_{i}\left(s_{1 i}, \ldots, s_{n i}\right)\right| \leq & \prod_{i=1}^{n} \sum_{\tau_{n i}=1}^{s_{n i}} \cdots \sum_{\tau_{1 i}=1}^{s_{1 i}}\left|\nabla_{n} \cdots \nabla_{1} a_{i}\left(\tau_{1 i}, \ldots, \tau_{n i}\right)\right| \\
\leq & \prod_{i=1}^{n}\left(s_{1 i} \cdots s_{n i}\right)^{1 / q_{i}}\left(\sum_{\tau_{n i}=1}^{s_{n i}} \cdots \sum_{\tau_{1 i}=1}^{s_{1 i}}\left|\nabla_{n} \cdots \nabla_{1} a_{i}\left(\tau_{1 i}, \ldots, \tau_{n i}\right)\right|^{p_{i}}\right)^{1 / p_{i}} \\
& \leq \frac{\left(\sum_{i=1}^{n}\left(s_{1 i} \cdots s_{n i}\right) / q_{i}\right)_{i=1}^{n} 1 / q_{i}}{\left(n-\sum_{i=1}^{n} 1 / p_{i}\right)^{n-\sum_{i=1}^{n} 1 / p_{i}}} \\
& \times \prod_{i=1}^{n}\left(\sum_{\tau_{n i}=1}^{s_{n i}} \cdots \sum_{\tau_{1 i}=1}^{s_{1 i}}\left|\nabla_{n} \cdots \nabla_{1} a_{i}\left(\tau_{1 i}, \ldots, \tau_{n i}\right)\right|^{p_{i}}\right)^{1 / p_{i}} .
\end{aligned}
$$

Dividing both sides of (3.2) by $\left(\sum_{i=1}^{n}\left(s_{1 i} \cdots s_{n i}\right) / q_{i}\right)^{\sum_{i=1}^{n} 1 / q_{i}}$ and then taking sums over $s_{j i}$ from 1 to $m_{j i}(i, j=1,2, \ldots, n)$, respectively and then using again Hölder's inequality, we obtain

$$
\begin{aligned}
\sum_{s_{11}=1}^{m_{11}} \cdots & \sum_{s_{n 1}=1}^{m_{n 1}} \sum_{s_{12}=1}^{m_{12}} \cdots \sum_{s_{n 2}=1}^{m_{n 2}} \cdots \sum_{s_{1 n}=1}^{m_{1 n}} \cdots \sum_{s_{n n}=1}^{m_{n n}} \frac{\prod_{i=1}^{n}\left|\nabla_{n} \cdots \nabla_{1} a_{i}\left(s_{1 i}, \ldots, s_{n i}\right)\right|}{\left(\sum_{i=1}^{n}\left(s_{1 i} \cdots s_{n i}\right) / q_{i}\right)^{\sum_{i=1}^{n} 1 / q_{i}}} \\
\leq & \left(n-\sum_{i=1}^{n} 1 / p_{i}\right)^{\sum_{i=1}^{n} 1 / p_{i}-n} \\
& \times \prod_{i=1}^{n}\left(\sum_{s_{n i}=1}^{m_{n i}} \cdots \sum_{s_{1 i}=1}^{m_{1 i}}\left(\sum_{\tau_{n i}=1}^{s_{n i}} \cdots \sum_{\tau_{1 i}=1}^{s_{1 i}}\left|\nabla_{n} \cdots \nabla_{1} a_{i}\left(\tau_{1 i}, \ldots, \tau_{n i}\right)\right|^{p_{i}}\right)^{1 / p_{i}}\right) \\
\leq & \left(n-\sum_{i=1}^{n} 1 / p_{i}\right)^{\sum_{i=1}^{n} 1 / p_{i}-n} \\
& \times \prod_{i=1}^{n}\left(m_{1 i} \cdots m_{n i}\right)^{1 / q_{i}}\left(\sum_{s_{n i}=1}^{m_{n i}} \cdots \sum_{s_{1 i}=1}^{m_{1 i}}\left(\sum_{\tau_{n i}=1}^{s_{n i}} \ldots \sum_{\tau_{1 i}=1}^{s_{1 i}}\left|\nabla_{n} \ldots \nabla_{1} a_{i}\left(\tau_{1 i}, \ldots, \tau_{n i}\right)\right|^{p_{i}}\right)\right)^{1 / p_{i}} \\
= & M \prod_{i=1}^{n}\left(\sum_{\tau_{n i}=1}^{m_{n i}} \cdots \sum_{\tau_{1 i}=1}^{m_{1 i}} \prod_{j=1}^{n}\left(m_{j i}-\tau_{j i}+1\right)\left|\nabla_{n} \cdots \nabla_{1} a_{i}\left(\tau_{1 i}, \ldots, \tau_{n i}\right)\right|^{p_{i}}\right)^{1 / p_{i}} \\
= & M \prod_{i=1}^{n}\left(\sum_{s_{n i}=1}^{m_{n i}} \cdots \sum_{s_{1 i}=1}^{m_{1 i}} \prod_{j=1}^{n}\left(m_{j i}-s_{j i}+1\right)\left|\nabla_{n} \cdots \nabla_{1} a_{i}\left(s_{1 i}, \ldots, s_{n i}\right)\right|^{p_{i}}\right)^{1 / p_{i}} .
\end{aligned}
$$

This concludes the proof. 
Proof of Theorem 2.2 From the hypotheses of Theorem 2.2, we have

$$
\left|f_{i}\left(x_{1 i}, \ldots, x_{n i}\right)\right| \leq \int_{0}^{x_{1 i}} \cdots \int_{0}^{x_{n i}}\left|\frac{\partial^{n}}{\partial \tau_{1 i} \cdots \partial \tau_{n i}} f_{i}\left(\tau_{1 i}, \ldots, \tau_{n i}\right)\right| d \tau_{1 i} \cdots d \tau_{n i} .
$$

On the other hand, by using Hölder's integral inequality (see [10]) and the following inequality for mean [10],

$$
\left(\prod_{i=1}^{n} \lambda_{i}^{1 / q_{i}}\right)^{1 / \sum_{i=1}^{n} 1 / q_{i}} \leq \frac{1}{\sum_{i=1}^{n} 1 / q_{i}} \sum_{i=1}^{n} \lambda_{i} / q_{i}, \quad \lambda_{i}>0
$$

we obtain

$$
\begin{aligned}
& \prod_{i=1}^{n}\left|f_{i}\left(x_{1 i}, \ldots, x_{n i}\right)\right| \\
& \leq \prod_{i=1}^{n} \int_{0}^{x_{1 i}} \cdots \int_{0}^{x_{n i}}\left|\frac{\partial^{n}}{\partial \tau_{1 i} \cdots \partial \tau_{n i}} f_{i}\left(\tau_{1 i}, \ldots, \tau_{n i}\right)\right| d \tau_{1 i} \cdots d \tau_{n i} \\
& \leq \prod_{i=1}^{n}\left(x_{1 i} \cdots x_{n i}\right)^{1 / q_{i}} \\
& \times\left(\int_{0}^{x_{1 i}} \cdots \int_{0}^{x_{n i}}\left|\frac{\partial^{n}}{\partial \tau_{1 i} \cdots \partial \tau_{n i}} f_{i}\left(\tau_{1 i}, \ldots, \tau_{n i}\right)\right|^{p_{i}} d \tau_{1 i} \cdots d \tau_{n i}\right)^{1 / p_{i}} \\
& \leq \frac{\left(\sum_{i=1}^{n}\left(x_{1 i} \cdots x_{n i}\right) / q_{i}\right)^{\sum_{i=1}^{n} 1 / q_{i}}}{\left(n-\sum_{i=1}^{n} 1 / p_{i}\right)^{n-\sum_{i=1}^{n} 1 / p_{i}}} \\
& \times \prod_{i=1}^{n}\left(\int_{0}^{x_{1 i}} \cdots \int_{0}^{x_{n i}}\left|\frac{\partial^{n}}{\partial \tau_{1 i} \cdots \partial \tau_{n i}} f_{i}\left(\tau_{1 i}, \ldots, \tau_{n i}\right)\right|^{p_{i}} d \tau_{1 i} \cdots d \tau_{n i}\right)^{1 / p_{i}} .
\end{aligned}
$$

Dividing both sides of (3.4) by $\left(\sum_{i=1}^{n}\left(x_{1 i} \cdots x_{n i}\right) / q_{i}\right)^{\sum_{i=1}^{n} 1 / q_{i}}$ and then integrating the result inequality over $x_{j i}$ from 1 to $t_{j i}(i, j=1,2, \ldots, n)$, respectively and then using again Hölder's integral inequality, we obtain

$$
\begin{aligned}
& \int_{0}^{t_{11}} \cdots \int_{0}^{t_{n 1}} \int_{0}^{t_{12}} \cdots \int_{0}^{t_{n 2}} \cdots \int_{0}^{t_{1 n}} \cdots \int_{0}^{t_{n n}} \\
& \quad \frac{\prod_{i=1}^{n}\left(\int_{0}^{x_{1 i}} \cdots \int_{0}^{x_{n i}}\left|\frac{\partial^{n}}{\partial \tau_{1 i} \cdots \partial \tau_{n l}} f_{i}\left(\tau_{1 i}, \ldots, \tau_{n i}\right)\right|^{p_{i}} d \tau_{1 i} \cdots d \tau_{n i}\right)^{1 / p_{i}}}{\left(\sum_{i=1}^{n}\left(x_{1 i} \cdots x_{n i}\right) / q_{i}\right)^{\sum_{i=1}^{n} 1 / q_{i}}} \\
& \leq\left(n-\sum_{i=1}^{n} 1 / p_{i}\right)^{\sum_{i=1}^{n} 1 / p_{i}-n} x_{n 1} d x_{12} \cdots d x_{n 2} \cdots d x_{1 n} \cdots d x_{n n} \\
& \times \prod_{i=1}^{n} \int_{0}^{t_{1 i}} \cdots \int_{0}^{t_{n i}}\left(\int_{0}^{x_{1 i}} \cdots \int_{0}^{x_{n i}}\right. \\
&\left.\left|\frac{\partial^{n}}{\partial \tau_{1 i} \cdots \partial \tau_{n i}} f_{i}\left(\tau_{1 i}, \ldots, \tau_{n i}\right)\right|^{p_{i}} d \tau_{1 i} \cdots d \tau_{n i}\right)^{1 / p_{i}} d x_{1 i} \cdots d x_{n i}
\end{aligned}
$$




$$
\begin{aligned}
\leq & \left(n-\sum_{i=1}^{n} 1 / p_{i}\right)^{\sum_{i=1}^{n} 1 / p_{i}-n} \prod_{i=1}^{n}\left(t_{1 i} \cdots t_{n i}\right)^{1 / q_{i}} \\
& \times\left(\int _ { 0 } ^ { t _ { 1 i } } \cdots \int _ { 0 } ^ { t _ { n i } } \left(\int_{0}^{x_{1 i}} \cdots \int_{0}^{x_{n i}}\right.\right. \\
& \left.\left.\left|\frac{\partial^{n}}{\partial \tau_{1 i} \cdots \partial \tau_{n i}} f_{i}\left(\tau_{1 i}, \ldots, \tau_{n i}\right)\right|^{p_{i}} d \tau_{1 i} \cdots d \tau_{n i}\right) d x_{1 i} \cdots d x_{n i}\right)^{1 / p_{i}} \\
= & N \prod_{i=1}^{n}\left(\int_{0}^{t_{1 i}} \cdots \int_{0}^{t_{n i}} \prod_{j=1}^{n}\left(t_{j i}-x_{j i}\right)\left|\frac{\partial^{n}}{\partial x_{1 i} \cdots \partial x_{n i}} f_{i}\left(x_{1 i}, \ldots, x_{n i}\right)\right|^{p_{i}} d x_{1 i} \cdots d x_{n i}\right)^{1 / p_{i}} .
\end{aligned}
$$

\section{This concludes the proof.}

\section{Competing interests}

The authors declare that they have no competing interests.

\section{Authors' contributions}

C-JZ and W-SC jointly contributed to the main results Theorems 2.1 and 2.2. All authors read and approved the final manuscript.

\section{Author details}

'Department of Mathematics, China Jiliang University, Hangzhou 310018, P.R. China. ${ }^{2}$ Department of Mathematics, The University of Hong Kong, Pokfulam Road, Hong Kong, P.R. China.

\section{Acknowledgement}

CJZ is supported by National Natural Science Foundation of China (10971205). WSC is partially supported by a HKU URG grant. The authors express their grateful thanks to the referees for their many very valuable suggestions and comments.

Received: 12 January 2012 Accepted: 6 June 2012 Published: 22 June 2012

\section{References}

1. Pachpatte, BG: On some new inequalities similar to Hilbert's inequality. J. Math. Anal. Appl. 226, 166-179 (1998)

2. Handley, GD, Koliha, JJ, Pečarić, JE: New Hilbert-Pachpatte type integral inequalities. J. Math. Anal. Appl. 257, 238-250 (2001)

3. Gao, MZ, Yang, BC: On the extended Hilbert's inequality. Proc. Am. Math. Soc. 126, 751-759 (1998)

4. Kuang, JC: On new extensions of Hilbert's integral inequality. J. Math. Anal. Appl. 235, 608-614 (1999)

5. Yang, BC: On new generalizations of Hilbert's inequality. J. Math. Anal. Appl. 248, 29-40 (2000)

6. Zhao, CJ: On inverses of disperse and continuous Pachpatte's inequalities. Acta Math. Sin. 46, 1111-1116 (2003)

7. Zhao, CJ: Generalizations on two new Hilbert type inequalities. J. Math. (Wuhan) 20, 413-416 (2000)

8. Zhao, CJ, Debnath, L: Some new inverse type Hilbert integral inequalities. J. Math. Anal. Appl. 262, 411-418 (2001)

9. Handley, GD, Koliha, JJ, Pečarić, JE: A Hilbert type inequality. Tamkang J. Math. 31, 311-315 (2000)

10. Hardy, GH, Littlewood, JE, Pólya, G: Inequalities. Cambridge University Press, Cambridge (1934)

11. Pachpatte, BG: Inequalities similar to certain extensions of Hilbert's inequality. J. Math. Anal. Appl. 243, $217-227$ (2000) 